\title{
Cost-effectiveness of monitoring glaucoma patients in shared care: an economic evaluation alongside a randomized controlled trial
}

\author{
Kim M Holtzer-Goor ${ }^{1 *}$, Esther van Sprundel ${ }^{2,3}$, Hans G Lemij ${ }^{3}$, Thomas Plochg ${ }^{4}$, Niek S Klazinga ${ }^{4}$, \\ Marc A Koopmanschap ${ }^{1}$
}

\begin{abstract}
Background: Population aging increases the number of glaucoma patients which leads to higher workloads of glaucoma specialists. If stable glaucoma patients were monitored by optometrists and ophthalmic technicians in a glaucoma follow-up unit (GFU) rather than by glaucoma specialists, the specialists' workload and waiting lists might be reduced.

We compared costs and quality of care at the GFU with those of usual care by glaucoma specialists in the Rotterdam Eye Hospital (REH) in a 30-month randomized clinical trial. Because quality of care turned out to be similar, we focus here on the costs.

Methods: Stable glaucoma patients were randomized between the GFU and the glaucoma specialist group. Costs per patient year were calculated from four perspectives: those of patients, the Rotterdam Eye Hospital (REH), Dutch healthcare system, and society. The outcome measures were: compliance to the protocol; patient satisfaction; stability according to the practitioner; mean difference in IOP; results of the examinations; and number of treatment changes.
\end{abstract}

Results: Baseline characteristics (such as age, intraocular pressure and target pressure) were comparable between the GFU group ( $n=410)$ and the glaucoma specialist group $(n=405)$.

Despite a higher number of visits per year, mean hospital costs per patient year were lower in the GFU group (€139 vs. €161). Patients' time and travel costs were similar. Healthcare costs were significantly lower for the GFU group (€230 vs. €251), as were societal costs (€310 vs. €339) ( $<<0.01)$. Bootstrap-, sensitivity- and scenario-analyses showed that the costs were robust when varying hospital policy and the duration of visits and tests.

Conclusion: We conclude that this GFU is cost-effective and deserves to be considered for implementation in other hospitals.

\section{Background}

Glaucoma is a group of eye diseases characterized by damage to the optic nerve that causes gradual, irreversible visual field loss. It is often related to high intraocular pressure (IOP) and age. Usual care for glaucoma patients consists of diagnosis, lifelong monitoring and treatment, and in most countries is currently provided by glaucoma specialists.

\footnotetext{
* Correspondence: goor@bmg.eur.nl

'Institute for Medical Technology Assessment-Erasmus University Rotterdam, Rotterdam, the Netherlands

Full list of author information is available at the end of the article
}

Ophthalmic care in the Netherlands is currently being challenged by a high workload for glaucoma specialists and long waiting lists. Due to ageing of the population, the prevalence of glaucoma probably will increase strongly over time [1], possibly endangering access to glaucoma care as currently provided. Task substitution may be one way to ease this problem.

Stable glaucoma patients and patients with a risk factor for developing glaucoma may not require care by a glaucoma specialist. Instead, monitoring by hospital optometrists or ophthalmic technicians may be sufficient. This would leave glaucoma specialists with more

\section{(Ciomed Central}


time for complex cases and new glaucoma patients, allocating their expertise more efficiently, and also reducing waiting lists. As optometrists and ophthalmic technicians are less expensive per hour than specialists, such task substitution might save costs.

To date, only one study [2-6] presented information about the efficiency of substitution in glaucoma care, and its consequences for both quality of care and costeffectiveness. However, that study compared care by glaucoma specialists with that by community optometrists rather than hospital optometrists. It concluded that glaucoma monitoring by community optometrists is effective, but not cost-effective in most situations. The outcomes were similar to those in specialist care, and the patients were satisfied. However, because of a (standard) shorter follow-up interval than in specialist care, community monitoring was more expensive [6].

We therefore conducted a randomized controlled trial (RCT) to determine the cost-effectiveness of shared care in stable glaucoma patients in a hospital setting. We compared usual care by glaucoma specialists and the care provided by optometrists and ophthalmic technicians within a glaucoma follow-up unit (GFU) in the Rotterdam Eye Hospital (REH) in terms of costs and quality of care.

Because this paper focuses on the costs of the glaucoma care related to important aspects of the quality of care, we also measured patient satisfaction, the number of treatment changes, the change in IOP and the compliance to the standard working protocol. The quality of care is described in more detail elsewhere. [7].

\section{Methods}

\section{Randomized Controlled Trial}

Patients who visited a glaucoma specialist or the GFU between September 2005 and April 2006 were invited to participate. The RCT was explained and written information was provided to them. The study was approved by the Review Board of Erasmus MC.

To be eligible for the study, patients had to meet the following criteria:

(1) the patient was diagnosed with stable glaucoma in one or both eyes (the next visit scheduled in 6 months or more) or had a risk factor for glaucoma, i.e. high IOP and/or a positive family history. Eyes were considered to be glaucomatous if they had typical thinning or notching of the neuroretinal rim of the optic nerve head, with or without disc haemorrhages, visual field defects, peripapillary atrophy and/or and elevated IOP;

(2) a glaucoma specialist of the REH referred the patient to the GFU;

(3) the actual ophthalmic medication and the target pressure (TP) was recorded in the medical record. The target pressure was determined by the individual clinicians in all patients, where they took in consideration: the age of the patient, the appearance of the optic disc, the level of intraocular pressure, any co-morbidity and any other risk factors. For patients with a risk factor for glaucoma, the TP was by default $30 \mathrm{mmHg}$, unless other risk factors called for an explicitly lower TP;

(4) an examination of the optic disc, macula, and the fundus periphery was performed;

(5) the Snellen visual acuity in each eye was $\geq 20 / 100$ and/or the patient had no visual field loss within the central $10^{\circ}$, as measured by a Humphrey Field Analyser, standard 24-2 test algorithm (HFA 24-2; Carl Zeiss Meditec, Dublin, CA, USA);

(6) the refractive error was between +5 and -8 dioptres (spherical equivalent);

(7) no other significant ocular disease was present;

(8) the patient had not undergone laser therapy for diabetic retinopathy.

Once the glaucoma specialist decided that the patient was suitable for the GFU, the patient was randomly allocated to a treatment group. In the glaucoma specialist group, the patients received care of glaucoma specialists and residents only. In the GFU group the patient visited the GFU twice followed by a visit to the glaucoma specialist or resident if the patient was stable. If necessary, the patient was seen by a glaucoma specialist earlier. The GFU employees (optometrist or ophthalmic technician level 1 or 2) provided care according to a standard working protocol (see Table 1) and under supervision of glaucoma specialists.

\section{Identification and Randomisation}

Glaucoma specialists were asked to provide information about this study to eligible patients. Eligible patients were also identified by searching the patient files of the patients that were already referred by the glaucoma specialists to the GFU in the months preceding the start of our study. They received information about the study during their next visit. All patients that were eligible and willing to participate were randomly allocated to a treatment group using a randomisation table. For the GFU patients that were allocated to the usual care group an appointment was made with the glaucoma specialist who referred them to the GFU.

To avoid glaucoma specialists influencing the allocation of patients, we used central randomisation by the researchers using stratification by 2 variables: the referring glaucoma specialist, and the time to the next scheduled visit, being either 6 months or more than 6 months.

\section{Outcome measures}

The outcome of the treatment was measured every visit. The outcome measures of the RCT were: 1) compliance 
Table 1 Provided care and criteria for back referral to the glaucoma specialist

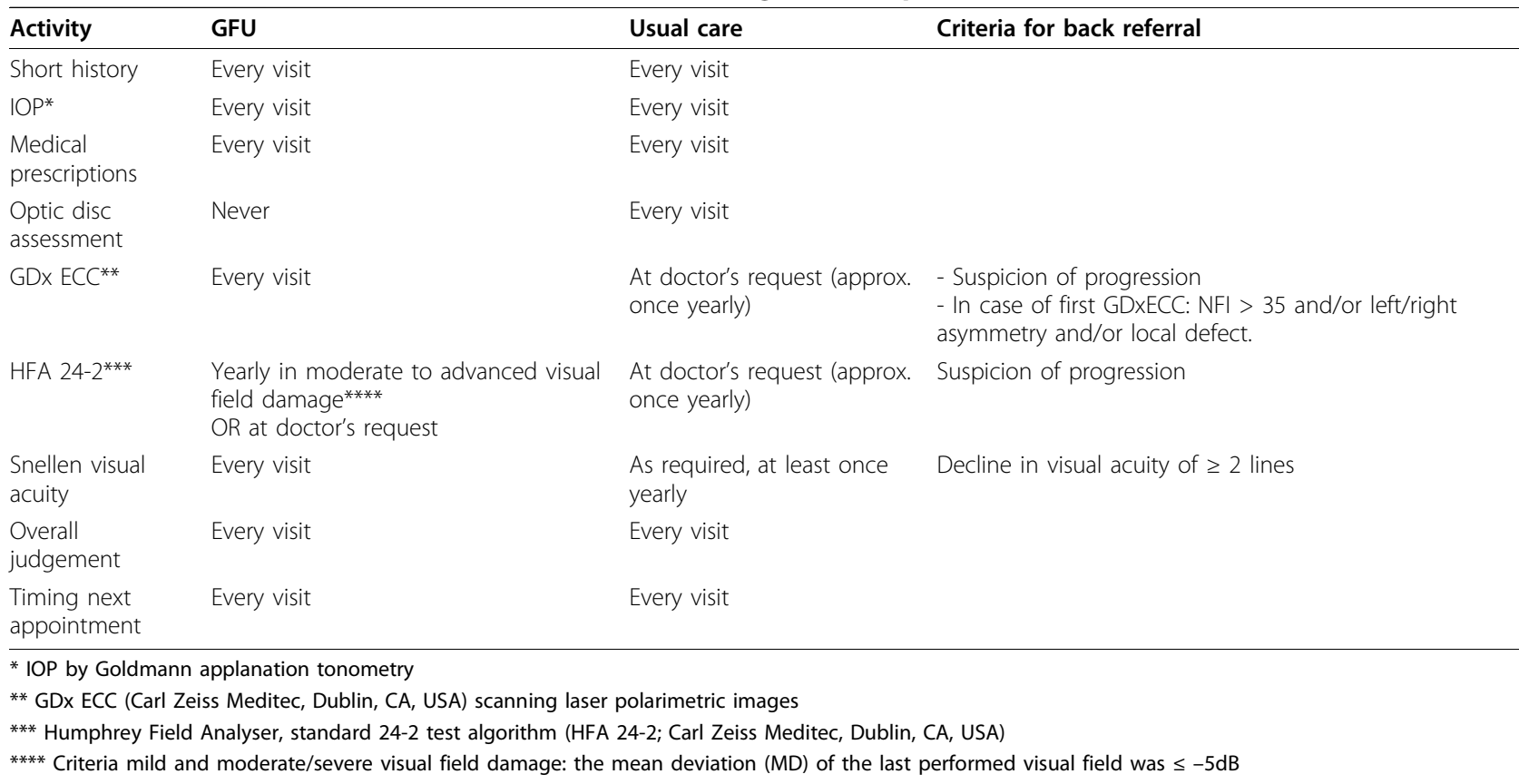

of the GFU employees to the standard working protocol, 2) patient satisfaction with the following items: a) overall mark for the received care; b) social interaction with the health care provider; c) expectations about the visit; d) perceived knowledge of the health care provider; e) waiting area, 3) stability according to the practitioner (whether the time till next visit should be significantly shorter than the time from the previous visit), 4) mean difference of the IOP (IOP at baseline vs. IOP at the last visit (if at least 24 months afterwards), 5) the results of the examinations and, 6) the number of treatment changes. We did not use glaucomatous progression as an outcome measure, because we did not expect this patient population (with a risk factor for glaucoma or with stable glaucoma) to progress during the study. The change in IOP during the study has been used as outcome measure instead.

\section{Sample size and power analysis}

We performed a post-hoc power analysis using our data to estimate the power (certainty) of our conclusion. We performed that analysis using two outcome parameters since quality of care has multiple dimensions: the stability of the patient according to the practitioner and the overall mark regarding patient satisfaction. The power of the study was $>99 \%$ based on the stability outcome when using $5 \%$ as an acceptable difference, and $>99 \%$ based on the overall mark when using a difference of 0.5 (on a 1-10 scale) as an acceptable difference between the treatment groups as well.

\section{Patients and visits}

From September 2005 to March 2006, 866 patients were included of which 46 patients did not visit the hospital during the study period. Three others could not be monitored with the GDx and 2 patients withdrew their informed consent (see Figure 1). The remaining 815 patients had a total of 2100 visits. The average time between visits was 8.8 months, $\mathrm{SD} \pm 4.0$. The mean age (63 years) and gender (53\% women) was similar for the two treatment groups. There were no significant clinical imbalances between the groups as well (Table 2).

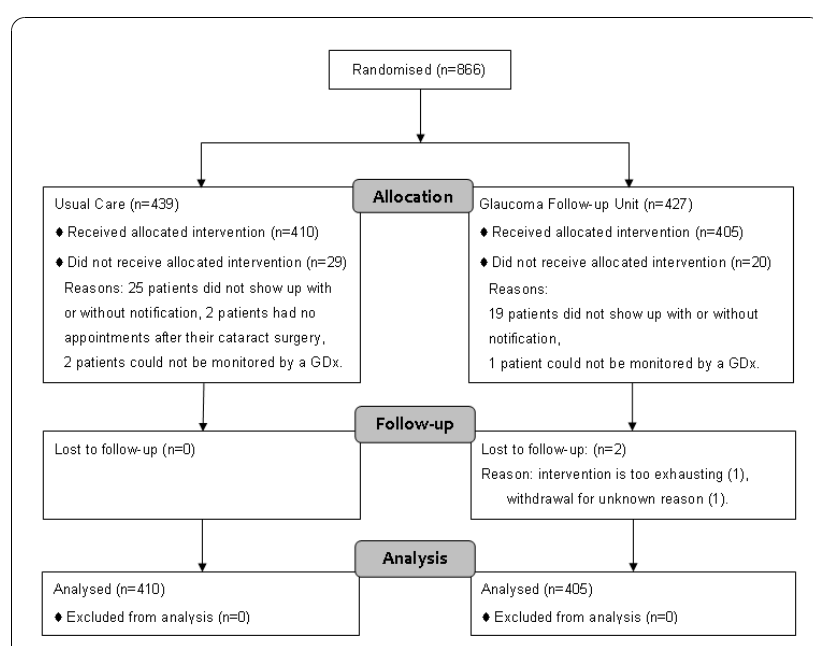

Figure 1 Patient flow chart. GDx $=$ Nerve Fiber Analyser. 
Table 2 Characteristics of included patients, by treatment group

\begin{tabular}{|c|c|c|c|c|}
\hline & \multicolumn{2}{|c|}{ Glaucoma Follow-up Unit $(n=405)$} & \multicolumn{2}{|c|}{ Usual Care $(n=410)$} \\
\hline Gender, \% of women & \multicolumn{2}{|c|}{53.8} & \multicolumn{2}{|c|}{52.2} \\
\hline Mean age (SD, standard deviation) & \multicolumn{2}{|c|}{$63.0(12.1)$} & \multicolumn{2}{|c|}{$63.1(11.9)$} \\
\hline \multirow[t]{2}{*}{ Mean time till next visit in months (SD) } & \multicolumn{2}{|c|}{$9.8(2.9)$} & \multicolumn{2}{|c|}{$9.5(2.9)$} \\
\hline & Right eye & Left eye & Right eye & Left eye \\
\hline Mean intraocular pressure (SD) & $18.7(4.1)$ & $18.5(4.1)$ & $18.8(4.2)$ & $18.8(4.1)$ \\
\hline Mean target intraocular pressure (SD) & $25.1(5.2)$ & $25.2(5.2)$ & $25.2(5.4)$ & $25.1(5.4)$ \\
\hline
\end{tabular}

$\mathrm{SD}=$ Standard Deviation

\section{Study duration}

The study duration depended on the allocated treatment group. Patients who were allocated to the usual care group on the day of their visit to the GFU, entered the study at their next visit (to the glaucoma specialist), whereas patients who were allocated to the GFU group, entered the study immediately. Therefore, the mean study duration was longer for the GFU group (1.81 year) than for the usual care group (1.43 year). This difference was statistically significant $(p<0.001)$. Hence, for a better comparison, we will present the costs per patient year in most tables. The influence of this difference in study duration on the outcomes is probably minimal, because no major changes were made to the protocol over time.

\section{Economic Evaluation}

We conducted an RCT to measure the quality of care delivered by glaucoma specialists and by employees of the GFU. Alongside this RCT, we calculated the costs of glaucoma care from four perspectives. The perspectives used were those of the patient, the REH, the health care system and the society.

A difference in health outcomes between the GFU and the usual care group was not expected during this study, because of the slowly progressive nature of this disease. A literature review, searching for articles with glaucoma and co-management or shared care in the title or abstract, provided evidence of an equal quality of care by optometrists compared to ophthalmologists as well. [3,4,8-18] Only one of the articles reported a variation in individual performances of optometrists, which makes education and accreditation an essential prerequisite for co-management. [10] All other articles reported good quality of care by optometrists, high levels of agreement between optometrists and a research clinic reference or ophthalmologists or comparable inter- and intra-observer variability in optic disc assessments. Therefore we will not present a cost-effectiveness ratio, but we will discuss the costs in relation to the quality of care.

\section{Identification of cost items and measurement of the utilization per cost item}

We interviewed health care professionals and patients to identify relevant cost items in the field of medical consumption, implementation of GFU, and patient time and travel costs.

During the RCT, the medical procedures performed and the medication prescribed each visit were recorded in a case report form. The different types of hospital visits were a visit to: a glaucoma specialist, a resident, and three types of GFU visits, as there were three different types of personnel within the GFU (optometrist, ophthalmic technician level 1 , and ophthalmic technician level 2). Per health care provider, the duration of 10 study related visits was measured. The duration of an HFA and GDx test were also measured in 10 patients.

Every visit, glaucoma patients were given a questionnaire to report their travelling distance, mode of transport, travelling time, waiting time and working status, in order to calculate the time and travelling costs. We also examined the fraction of visits in which the GFU employees asked a glaucoma specialist for advice over time. In addition, we performed a logistic regression to determine which variables influenced the probability of asking advice.

The substitution of care to the GFU required organizational changes and hence implementation costs (both initial and structural) within the hospital. To collect this information, health care providers were interviewed.

\section{Valuation of the cost items}

All costs were calculated (in euros, price level 2007) according to the CVZ (The Health Care Insurance Board) costing guidelines and previous research in the REH.

[19] Relevant items from the CVZ costing guidelines $[20,21]$ were updated and used for the calculation of patient time costs per hour and travelling costs per kilometer.

Our cost calculation of hospital costs is based on data from the internal budget allocation provided by the 
REH financial administration. This information included location costs, costs of medical specialists and other personnel, administrative costs, costs of equipment, overhead costs and interest. Only for the costs of non-laser operations was the $\mathrm{DBC}$ rate (Diagnosis Treatment combination- a fixed reimbursement rate for a specific diagnosis related therapy) in 2007 used as estimate of the resource costs.

The direct personnel costs were calculated based on the mean duration for each type of visit. However, the indirect personnel and overhead costs were calculated top-down, based on the mean duration of a visit in the hospital as a whole.

The implementation costs, like internal preparatory meetings, visits to another Dutch hospital, writing the standard working protocol and the training of the employees of the GFU were dominated by personnel input. These costs were added to the costs of a GFU visit as implementation costs for the GFU. The initial implementation costs that were only made before starting the GFU were spread over 5 years. The structural costs per year were added to the initial implementation costs per year. The implementation costs per visit were based on the total number of GFU visits in 2007 (1598 visits) as we expect this number of patients to be a representation of the number of patients in the near future.

We calculated the patient costs using the information of the patient questionnaires combined with the updated time and travelling costs per unit of time and per kilometer. The results will be expressed as average costs per patient per study year and average costs per patient.

\section{Sensitivity/scenario analysis}

To determine the influence of uncertainty regarding the duration of visits or tests on the costs per patient year, we performed the following uni-variate sensitivity analyses:

1. We varied the duration of the visits within the range we had measured in our study. This resulted in 4 scenarios:

a. We used the minimum duration for all visits;

b. We used the maximum duration for all visits;

c. We used the minimum duration of visits to the GFU and the maximum duration for the visits to the glaucoma specialist and resident;

$\mathrm{d}$. We used the maximum duration of visits to the GFU and the minimum duration for the visits to the glaucoma specialist and resident.

2. We used the norm duration of the GDx and HFA as used by the financial department, instead of the duration of the GDx and HFA measured in our study.
Furthermore we performed scenario analyses to determine the effects of plausible policy changes in the (near) future on the costs. We considered the following scenarios:

3. No optometrists are working in the GFU. This actually happened during the course of the study. The direct personnel costs of visits to optometrists were replaced by those of the ophthalmic technicians.

4. In the study, the patients in the GFU group visited the glaucoma specialist (or resident) every third visit, or earlier if necessary. In this scenario, we calculated the visit costs if this routine was changed to every fifth visit (or earlier when necessary). In case of a non stable patient, we distinguished two scenarios:

a. The patient returned to the GFU as soon as he was judged as stable by the glaucoma specialist during a visit.

b. The patient only returned to the GFU after he was judged as stable by the glaucoma specialist on two consecutive visits.

\section{Uncertainty analysis}

We performed a bootstrapping analysis on the costs per patient year and two quality of care parameters, to show the degree of uncertainty regarding the results. Since quality of care has different dimensions, we decided to use two outcome parameters. One clinical quality parameter: stability according to the practitioner (stability), and one patient satisfaction parameter: the overall mark given by the patient. By plotting all bootstrap replicates in a so-called cost-effectiveness plane (CE-plane), the uncertainty around the point estimates of the costs and effects was displayed. In this analysis individual observations of patients were randomly drawn from the distribution of patients in both groups in order to calculate the average costs and quality of care per treatment group. This was replicated for 2500 times. A CE-plane is an $\mathrm{x}$-y-diagram with the $\mathrm{x}$-axis representing the difference in quality of care between the GFU and usual care group and the y-axis representing the difference in costs.

\section{Statistical analysis}

We used Excel for the bootstrapping analysis. SPSS 15.0 was used for all other analyses. In normally distributed variables, we performed a t-test for independent samples. If not distributed normally, we performed the parametric Mann-Whitney U-test to compare the two treatment groups. We used bootstrapping for deriving the $95 \%$ confidence intervals around the utilization and costs because of the non-normal distribution of those parameters. 
For some visits (29\%), information about one or more items related to patient costs was missing. The travelling distance could be calculated for every patient, based on the Zip code as known in the hospital information system. If appropriate, the remaining missing values were replaced by values known from other visits of the same patient. In all other cases (9\%), the mean value of a comparable group of patients based on gender and age was imputed to the missing values.

\section{Results}

\section{Quality of care}

The aspects of quality of care measured in our study were: compliance to the protocol, patient satisfaction, stability according to the practitioner, mean difference of the IOP, results of the examinations and the number of treatment changes. All these aspects of the quality of care turned out to be similar for the 2 groups (see also reference 7) and the substitution of care to the GFU was successfully implemented.

1. The GFU employees performed the required tests in at least $98.8 \%$ of the visits and referred back to the glaucoma specialist in $84.4 \%$ of the remarkable cases. 2 . The patient satisfaction was similar in both groups. The overall mark of the patient was 8.5 for the GFU group and 8.4 for the usual care group $(\mathrm{p}=0.147)$.

3 . The percentage of visits that were considered "stable" was $16 \%$ in the usual care group and $17 \%$ in the GFU group ( $p=0.423$ )

4. No statistical difference was found between the two groups in the difference of the IOP during the study (IOP ( $\geq 24$ months since baseline) $-I O P$ (at baseline)).

5 . The average difference in IOP OD was $-0.2 \mathrm{mmHG}$ in the usual care group and $-0.6 \mathrm{mmHG}$ in the GFU treatment group $(\mathrm{p}=0.207)$. The average difference in IOP OS was $-0.1 \mathrm{mmHg}$ in both groups $(\mathrm{p}=0.915)$.

6. The number of treatment changes was 57 (14\%) in the GFU group and $63(15 \%)$ in the usual care group ( $\mathrm{p}=0.603$ ).

7. Patients as well as GFU employees and glaucoma specialists were pleased with the functioning of the GFU.

Therefore, the quality of care provided in the GFU was concluded to be equal to the care provided by the glaucoma specialists for these stable glaucoma patients.

\section{Hospital perspective}

The hospital costs covered hospital visits, diagnostic procedures and further treatment, but were mainly driven by the costs of the hospital visits to the glaucoma specialist, resident or GFU employee (approximately $80 \%)$. Table 3 shows the duration and composition of the unit costs per type of visit. The total annual implementation costs for starting up the GFU were $€ 4917$ for 1598 GFU visits. The implementation costs of the GFU were added to the GFU visits only.

Table 3 shows that despite their longer duration, GFU visits were less expensive than those to the glaucoma specialist. In the usual care group, most visits were paid to the glaucoma specialist or resident. Patients in the GFU group visited the glaucoma specialist every third visit or earlier when a patient was judged not stable. Therefore, the costs per visit could vary within one patient and between patients within one treatment group. The mean costs per hospital visit including GDx were $€ 83.77$ ( $S D=30.64)$ in the usual care group and $€ 68.34$ (SD = 15.66) in the GFU group. This difference was statistically significant (t-test, $\mathrm{p}=0.000$ ).

Table 4 describes the hospital care use per patient year for the two treatment groups. Although the number of visits per patient year was slightly higher in the GFU group (1.65 vs. 1.57), this difference was not statistically significant. In the GFU group, a significantly larger number of GDx images (1.28 vs. 0.77$)$ and autorefractions ( 0.20 vs. 0.08$)$ was performed and more time was spent on asking advice (in $24 \%$ vs. $10 \%$ of the visits). On the other hand, glaucoma surgery, laser therapy, medication use and the number of HFA tests did not statistically differ between the two groups.

The hospital care use has been translated into costs per patient year for the two treatment groups in Table 5. The total hospital costs were significantly higher for the usual care group than for the GFU group, mainly because of the higher hospital visit costs. The costs of asking advice were modest, but significantly higher for the GFU group than for the usual care group, as was to be expected. The $95 \%$ confidence interval for the difference in total hospital costs as derived from the bootstrap analysis was $€-59$ to $€-2$. The probability that the GFU reduces hospital costs was $98 \%$.

The proportion of visits by GFU employees needing advice increased initially from $15 \%$ to $20 \%$ in 2006 and then decreased (statistically significant) to $13 \%$ in 2007 and $7 \%$ in 2008 . The proportion of visits requiring advice was not affected by the total number of visits per patient.

These findings were confirmed by a logistic regression. The year of the visit was the only variable that significantly influenced the probability of asking advice. The other variables in the regression analysis were: stable/ not stable, visit number, gender, time till next visit and age. This indicates that the GFU employees got more experienced over time and therefore needed less advice.

\section{Patient perspective}

The patient costs consisted of time and travelling costs of patients and their accompaniment. Table 5 shows 
Table 3 The composition of the unit costs per type of hospital visit in $€$ (2007)

\begin{tabular}{llllll}
\hline & $\begin{array}{l}\text { Visit } \\
\text { glaucoma specialist }\end{array}$ & $\begin{array}{l}\text { Visit } \\
\text { resident }\end{array}$ & $\begin{array}{l}\text { Visit GFU } \\
\text { Optometrist* }\end{array}$ & $\begin{array}{l}\text { Visit GFU } \\
\text { TOA level } \\
\mathbf{1}^{* * *}\end{array}$ & $\begin{array}{l}\text { Visit GFU } \\
\text { TOA level } \\
\mathbf{2}^{* * * *}\end{array}$ \\
\hline Costs per visit & & & & 15.05 & 16.61 \\
Total direct personnel costs & 24.36 & 14.49 & 19.09 & 6.59 & 6.59 \\
Total indirect personnel costs & 5.46 & 5.46 & 6.59 & 35.90 & 35.90 \\
Total overhead costs & 29.76 & 29.76 & 35.90 & 3.08 & 3.08 \\
Implementation costs GFU & 0.00 & 0.00 & 3.08 & 60.62 & 62.18 \\
\hline Total costs excluding GDx & 59.58 & 49.71 & 64.66 & 3.05 & 3.05 \\
Costs GDx*** (fraction performed) & $25.00(0.41)$ & $22.25(0.36)$ & 3.05 & $\mathbf{6 3 . 6 7}$ & $\mathbf{6 5 . 2 3}$ \\
\hline Total costs including GDx & $\mathbf{8 4 . 5 8}$ & $\mathbf{7 1 . 9 6}$ & $\mathbf{6 7 . 7 1}$ & 20.40 & 20.40 \\
Mean visit duration (min) & 9.06 & 11.00 & 20.40 & & \\
\hline
\end{tabular}

*Visit to an optometrist or senior employee

**Visit to an ophthalmic technician level 1

***Visit to an ophthalmic technician level 2

${ }^{* * * *}$ At the start of the Glaucoma Follow up unit (GFU), a Nerve Fiber Analyser (GDx) was purchased by the Rotterdam Eye Hospital (REH). The costs of the GDx performed during GFU visits, consists only of the GDx imaging device. In the usual care group, the GDx was performed during an extra visit to the perimetry department. In that situation, the costs of a GDx image included personnel and overhead costs as well and were $€ 61.61$ based on a duration of 13.30 minutes

that the patient costs per visit were significantly higher in the usual care group, because of higher time costs ( $€ 80$ vs. $€ 88$ ). This was mainly caused by a longer waiting time in the hospital in the usual care group. Patients in the GFU group spent, on average, 44.6 minutes in the hospital against 59.4 minutes for the patients in usual care group $(p=0.000)$. However, because of a higher number of visits per patient year in the GFU arm, the patient costs per patient year were not statistically significantly higher anymore. The $95 \%$ confidence interval based on the bootstrapping analysis confirmed this ( $€-29$ to $€ 12)$. However, there is still a $78 \%$ probability to reduce patient costs.

\section{Health care perspective}

The health care costs consisted of the hospital costs as described above, and of medication costs. Table 5 shows the health care costs per patient year. Because of the comparable medication costs and lower hospital costs in the GFU group, the total health care costs per patient year were nearly $10 \%$ lower for the GFU group ( $€ 230.39$ vs. $€ 251.26, \mathrm{p}=0.04)$. The median cost differ statistically according to the Mann-Whitney U-test, but the confidence interval as provided through bootstrapping does not show a difference in the mean costs $(€-76$ to $€ 21)$. However, the probability of cost reduction is considerable: $87 \%$.

Table 4 Average hospital care use per patient year for the two treatment groups

\begin{tabular}{|c|c|c|c|c|c|}
\hline & GFU & $\begin{array}{l}\text { Usual } \\
\text { care }\end{array}$ & $\begin{array}{l}95 \%-\mathrm{Cl} \text { of } \\
\text { difference } \\
\text { between } 2 \\
\text { groups }\end{array}$ & P-value & $\begin{array}{l}\text { Costs per unit } \\
\text { (in } € \text { ) }\end{array}$ \\
\hline Hospital visits & 1.65 & 1.57 & -0.13 to +0.31 & 0.158 & See Table 2 \\
\hline GDx ECC & 1.28 & 0.77 & +0.32 to +0.73 & 0.000 & 61.61 \\
\hline HFA & 0.10 & 0.11 & -0.11 to +0.07 & 0.266 & 158.44 \\
\hline Refractive Unit & 0.01 & 0.05 & -0.09 to +0.00 & 0.002 & 32.43 \\
\hline Auto-refraction & 0.20 & 0.08 & -0.03 to +0.21 & 0.000 & $4.64-6.59^{*}$ \\
\hline Pachymetry & 0.02 & 0.04 & -0.07 to +0.03 & 0.246 & 23.17 \\
\hline IOP diurnal curve & 0.01 & 0.02 & -0.04 to +0.01 & 0.109 & 92.66 \\
\hline Laser treatment & 0.002 & 0.007 & -0.02 to +0.01 & 0.267 & 78.38 \\
\hline Glaucoma surgery & 0.002 & 0.001 & -0.01 to +0.01 & 0.558 & 1251.70 \\
\hline Asking advice & 0.24 & 0.10 & +0.05 to +0.26 & 0.000 & $8.19-15.86^{* *}$ \\
\hline Proportion patients using medication & 0.57 & 0.59 & -0.17 to +0.15 & 0.614 & $2.53-18.82^{* * *}$ \\
\hline
\end{tabular}

GFU = Glaucoma Follow-up Unit; Cl = confidence interval; GDx ECC = Nerve Fiber Analyser Enhanced Corneal Compensation; HFA = Humphrey Field Analyser; $\mathrm{IOP}=$ Intra Ocular Pressure

*Depending on health care provider

${ }^{* *}$ Costs per advice, depending on the health care providers involved

***Costs per month 
Table 5 Average costs in Euros per patient year per perspective used for the two treatment groups (SD)

\begin{tabular}{|c|c|c|c|}
\hline & GFU & Usual Care & P-value \\
\hline \multicolumn{4}{|l|}{ Hospital perspective } \\
\hline Hospital visits (including GDx ECC) & $111.93(50.93)$ & $133.17(50.44)$ & 0.000 \\
\hline Other tests (HFA, refraction, pachymetry, etc.) & $20.66(47.03)$ & $24.18(48.72)$ & 0.001 \\
\hline Laser treatment related to glaucoma & $0.18(2.54)$ & $0.57(5.18)$ & 0.258 \\
\hline Glaucoma surgery & $2.84(40.35)$ & $1.72(34.90)$ & 0.558 \\
\hline Asking advice & $3.24(5.35)$ & $1.78(5.13)$ & 0.000 \\
\hline Total hospital costs per patient year & $138.85(89.30)$ & $161.43(86.88)$ & 0.000 \\
\hline \multicolumn{4}{|l|}{ Patient perspective } \\
\hline \multicolumn{4}{|l|}{ Patient costs per visit } \\
\hline Travelling costs of patient and accompaniment & $8.26(11.83)$ & $8.19(12.10)$ & 0.966 \\
\hline Time costs of patient and accompaniment & $40.58(28.87)$ & $47.51(34.36)$ & 0.000 \\
\hline Total patient costs per patient per visit & $48.83(33.68)$ & $55.70(37.88)$ & 0.000 \\
\hline \multicolumn{4}{|l|}{ Patient costs per patient year } \\
\hline Travelling costs of patient and accompaniment & $13.04(17.16)$ & $12.70(17.87)$ & 0.488 \\
\hline Time costs of patient and accompaniment & $66.62(50.20)$ & $75.17(61.37)$ & 0.088 \\
\hline Total patient costs per patient year & $79.66(58.51)$ & $87.87(68.17)$ & 0.143 \\
\hline \multicolumn{4}{|l|}{ Health care perspective } \\
\hline Hospital costs & $138.85(89.30)$ & $161.43(86.88)$ & 0.000 \\
\hline Medication costs & $91.54(101.37)$ & $89.82(100.53)$ & 0.867 \\
\hline Total health care costs per patient year & $230.39(154.57)$ & $251.26(146.02)$ & 0.004 \\
\hline \multicolumn{4}{|l|}{ Societal perspective } \\
\hline Hospital costs & $138.85(89.30)$ & $161.43(86.88)$ & 0.000 \\
\hline Patient costs & $79.66(58.51)$ & $87.87(68.15)$ & 0.143 \\
\hline Medication costs & $91.54(101.37)$ & $89.82(100.53)$ & 0.867 \\
\hline Total societal costs per patient year & $310.05(181.86)$ & $339.13(180.39)$ & 0.009 \\
\hline
\end{tabular}

SD = Standard Deviation; GFU = Glaucoma Follow-up Unit; GDx ECC = Nerve Fiber Analyser Enhanced Corneal Compensation; HFA = Humphrey Field Analyser.

\section{Societal perspective}

In the societal perspective all costs were taken into account. It consisted of hospital costs, medication and patient costs, for $46 \%, 28 \%$ and $26 \%$ respectively. The total societal costs per patient year were almost $10 \%$ higher in the usual care group (Table 5: $€ 339.13$ vs. $€ 310.05, \mathrm{p}=0.009$ ). The mean difference in the total societal costs per patient year was $€-36$ (the GFU group was less expensive). The 95\% confidence interval based on bootstrapping for this difference ranged from $€-92$ to $€ 23$. Thus, though the median costs per patient year differs between the two groups, the mean total costs are not statistically different. This is because the non-normal distribution of the societal costs. However, the probability that the GFU saves societal costs is $84 \%$ to $89 \%$ (see paragraph about the uncertainty analysis below).

\section{Sensitivity/scenario analysis}

\section{Analysis 1: duration of visit}

The mean duration of the visits to the glaucoma specialist was 9 minutes (ranging from 7 to 11 minutes), to the resident 11 minutes (ranging from 9 to 13 minutes) and to the GFU 20 minutes (ranging from 16 to 24 minutes).
In the base case - the situation as in our study -, the GFU group was less expensive than the usual care group. This conclusion only changed when the duration of a visit in the usual care group would be relatively short (7 minutes) and the duration of a visit in the GFU group would be relatively long (24 minutes, scenario $1 d)$. In that unlikely situation the hospital costs per patient year were 10\% higher for the GFU group (see Table 6).

\section{Analysis 2: duration HFA/GDX}

The norm durations of GDx and HFA as used by the financial department, were 15 and 45 minutes respectively instead of 13.30 and 34.20 minutes. This longer duration of the HFA and GDx tests increased the hospital costs per patient year in the GFU group and usual care group with $€ 15$ and $€ 12$ respectively (Table 6).

\section{Analysis 3: no optometrist in GFU}

When the direct personnel costs of the optometrist were replaced by those of the ophthalmic technicians, the costs in both groups decreased, because incidentally a visit was paid to an optometrist in the usual care group as well (Table 6). Although the decrease in costs per patient year is small, it is almost twice as high in the 
Table 6 Total average hospital costs per patient year in Euros for all situations in the sensitivity/scenario analysis

\begin{tabular}{lcc}
\hline & \multicolumn{2}{c}{ Hospital costs } \\
& Usual care \\
\hline Base case & 138.85 & 161.43 \\
Scenario 1a & 117.79 & 141.80 \\
Scenario 1b & 156.79 & 179.98 \\
Scenario 1c & 117.79 & 179.98 \\
Scenario 1d & 156.79 & 141.80 \\
Scenario 2 & 153.78 & 173.18 \\
Scenario 3 & 135.16 & 159.47 \\
\hline
\end{tabular}

GFU group (€3.69) as in the usual care group (€1.96). Thus, the costs remain lower for the GFU group.

\section{Analysis 4: fewer specialist visits in GFU}

a. In this scenario, the total savings were $€ 2193$ for five visits of 427 patients. Based on a mean number of 1.65 visits per year as measured in this study, the hospital costs could be reduced with $€ 1.69$ per patient year.

b. In the second scenario, the total savings in visit costs were $€ 1882$ for five visits of 427 patients, thereby reducing the hospital costs in the GFU group with $€ 1.45$ per patient year $(=1 \%)$.

\section{Uncertainty analysis}

From a societal perspective, the incremental cost-effectiveness ratio of the GFU compared with usual care was - €27 per patient per decimal point increase of the patients' overall mark (on a 1-10 scale) per year. The CE-plane with overall mark as outcome showed that the majority of bootstrap replications (70\%) fell within the lower-right quadrant, indicating that the GFU was dominant with lower costs and a higher overall mark (Figure 2a).

The incremental cost-effectiveness ratio for the GFU compared with usual care was $+€ 19$ per patient per year for one extra percent of visits that were considered to be stable by the practitioner. For the CE-plane with "stability" as outcome, the majority of bootstrap replications fell within the lower-left quadrant which reflects lower costs and fewer stable visits (Figure $2 \mathrm{~b}$ ). The probability that the GFU is cost saving is $89 \%$ using the overall mark and $84 \%$ using the "stability" outcome. Against this high probability of saving costs, the probability of inferiority of the GFU (being more expensive and less effective) is quite small: $2 \%$ using the overall mark and $14 \%$ using the "stability" outcome.

Using an acceptable difference of 0.5 point of the overall mark (range 1-10), and of 5\% difference in the fraction of stable patients, the two groups have an equal quality of care in $99.5 \%$ and $80.5 \%$ of the bootstrap replications respectively. When including replications that result in a better quality of life for the GFU, the quality of care is acceptable (equal or better) in 100\% and in $83.6 \%$ of the bootstrap replications.

\section{Discussion}

Substitution of tasks that require less specialized skills is a possible solution for easing the increased workload of ophthalmologists and long waiting lists in ophthalmic care. It was hypothesized that task substitution reduces the costs as well. The monitoring of most stable glaucoma patients probably does not require specialized skills. In this study, we therefore compared the care as usual provided by glaucoma specialists with the care provided by a GFU within the REH staffed by ophthalmic technicians and optometrists for stable glaucoma patients.

We found about $10 \%$ lower health care costs per patient year for the GFU group compared to the usual care group for three of the four perspectives used: the $\mathrm{REH}$, the health care system and the society. Patient costs did not differ between the two treatment groups.

Scenario and sensitivity analyses confirmed that our results were robust. Only if the mean duration of a visit increased in the GFU (with $18 \%$ to the maximum duration measured in this study) and decreased for the glaucoma specialist (with $23 \%$ to the minimum duration measured in our study, scenario 1d), would the total societal costs not be significantly different any longer. However, this situation is not realistic. The bootstrap analysis showed that the equivalence of the two groups on quality of care is justified and that the GFU is cost saving in $89 \%$ of the bootstrap replicates when using the overall mark as outcome parameter and in $84 \%$ of the replicates when using the stability of the patient according to the practitioner.

We hypothesized that the establishment of the GFU would reduce the waiting list. This was confirmed by the increased number of patients $(+23 \%)$ and patient visits $(+16 \%)$ per year within the study period. The increased number of visits was largely caused by the establishment of the GFU, whereas the rise in the number of glaucoma patients was also influenced a little by a reduced follow-up interval for some glaucoma patients. However, the long term effect on the waiting list seems to be limited. Possible causes are: the chronic character of the disease which limits the patient outflow and the substantial increase in new glaucoma patients that outweighs the growth in capacity. Further research would be necessary to explore the true cause(s).

The hospital perspective was one of the perspectives used for the cost calculation. Although the probability that the GFU is cost-effective from this perspective is 94-98\%, we have to distinguish at least two stakeholders within the hospital; the hospital management and the glaucoma specialists. The interests of those two 

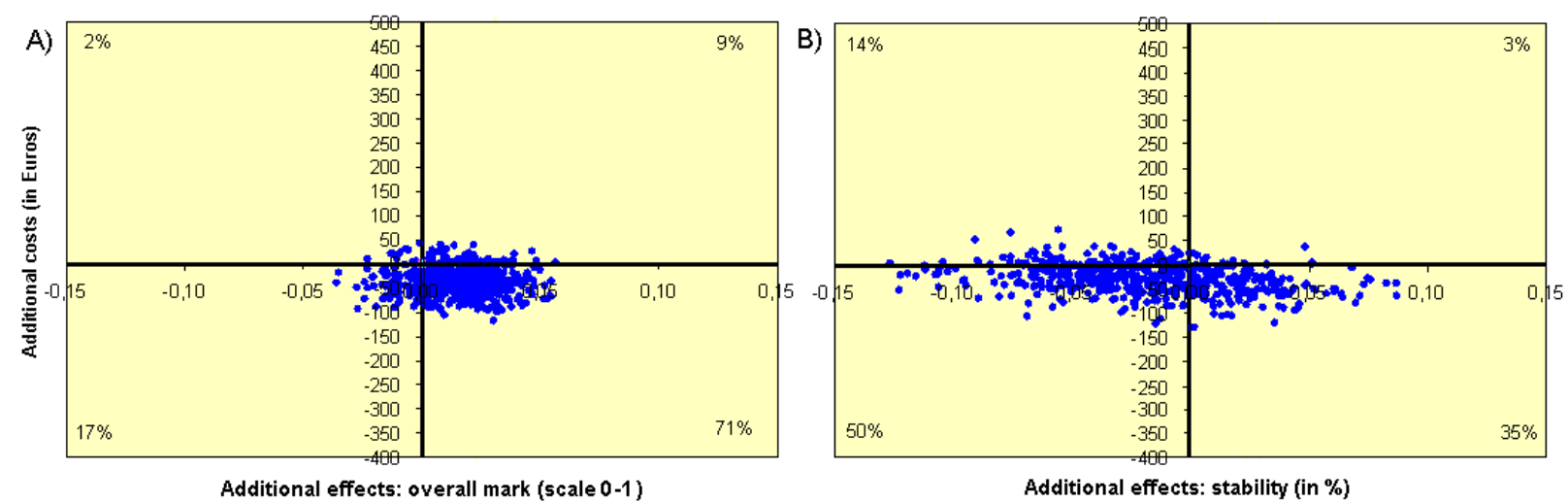

Figure 2 Cost-effectiveness plane. A) yearly incremental costs per patient vs. change in the patients' overall mark. B) yearly incremental costs per patient vs. change in the percentage of stable visits.

stakeholders are partially conflicting due to the current structure of financing care in the Netherlands. The physician part of the reimbursement is now paid to the specialist although the monitoring is partially transferred to the GFU. The distribution of this fee will therefore become subject of discussion between glaucoma specialists and the hospital management, especially when health care insurers insist on a lower fee in future negotiations, because of the lower costs of monitoring glaucoma patients by the GFU.

Our results could not be easily compared with results of other research. Even though substituting tasks within the hospital setting is taking place, a full cost calculation of this kind of substitution in the ophthalmic care has not been performed yet. In Bristol (UK), an economic evaluation alongside an RCT has been performed, comparing costs of monitoring stable glaucoma patients by ophthalmologists and community optometrists (outside the hospital). [2,6] Contrary to ours, the UK study concluded that the substitution of care to community optometrists was not likely to save costs. The main reason for this was the larger number of referrals to the ophthalmologist in their study compared to ours $(19 \%$ vs. $6 \%$ ). An explanation for this difference might be the location of care. Community optometrists do not have the possibility to consult a specialist for quick advice and will therefore refer patients to the hospital relatively more often.

Furthermore, the frequency of visits to the community optometrist in Bristol was $66 \%$ higher than the visit frequency to the ophthalmologist, compared with a $5 \%$ higher frequency in our study. This difference is related to a difference in the protocol used. In our study, the time to the next visit was copied from the last visit to the glaucoma specialist instead of being pre-determined at 6 months.
A study about the trends in outpatient care provided by physicians and non-physician clinicians showed that substitution of care is not always a good strategy for containing health care costs. [22] The increase in the proportion of patients visiting a non-physician clinician is driven by the increase in patients visiting both a nonphysician and a physician clinician. In our study however, the number of extra visits caused by referrals was relatively low as stated earlier.

A possible drawback of our study is the lack of information about disease progression. The progression rate of glaucoma depends on the intraocular pressure, and the time to vision loss varies between 3 years for untreated patients with a high intraocular pressure to 38 years for well treated patients. [23,24] We therefore did not expect to detect any significant glaucomatous progression in the 30-months study period in these stable patients and performed a cost minimization study. This type of economic evaluation assumes an equal outcome for all patients. The results of the RCT [7] as well as many other studies $[3,4,8-18]$ supported this assumption about the equal quality of care to glaucoma patients provided by different types of health care providers.

\section{Conclusions}

Considering the equal quality of care in both treatment groups, we conclude that monitoring of glaucoma patients by the GFU is cost-effective for a subset of glaucoma patients, i.e., those that were deemed stable in the Rotterdam Eye Hospital. Implementation of a similar GFU in other hospitals could therefore be considered.

\section{Acknowledgements}

We thank Els Steijger and Ilse Nederveen for the REH cost information. The study has been funded by ZonMW (The Netherlands organization for health research and development), and The Rotterdam Eye Hospital 
Foundation Prof. Dr. H.J. Flieringa. It was approved by the Review Board of Erasmus MC

The funding organizations had no role in the study design, in the writing of the manuscript or the decision to submit the manuscript for publication.

\section{Author details}

1 Institute for Medical Technology Assessment-Erasmus University Rotterdam, Rotterdam, the Netherlands. ${ }^{2}$ Rotterdam Ophthalmic Institute, Rotterdam, the Netherlands. ${ }^{3}$ The Rotterdam Eye Hospital, Rotterdam, the Netherlands. ${ }^{4}$ Department of Social Medicine, Academic Medical Center (AMC)/University of Amsterdam, the Netherlands.

\section{Authors' contributions}

KMHG carried out the cost calculations and drafted the manuscript. EvS surveyed the resource use of the patients, and drafted the manuscript. HGL was involved in setting up the Glaucoma Follow-up Unit and reviewed the manuscript. TP and NSK participated in the design of the study and reviewed the manuscript. MAK participated in the design and coordination of the study and helped to draft the manuscript. All authors interpreted the results, read and approved the final manuscript.

\section{Competing interests}

The authors declare that they have no competing interests.

Received: 12 February 2010 Accepted: 17 November 2010 Published: 17 November 2010

\section{References}

1. Quigley HA, Broman AT: The number of people with glaucoma worldwide in 2010 and 2020. Br J Ophthalmol 2006, 90:262-7.

2. Coast J, Spencer IC, Smith L, Spry PG: Comparing costs of monitoring glaucoma patients: hospital ophthalmologists versus community optometrists. J Health Serv Res Policy 1997, 2:19-25.

3. Gray SF, Spencer IC, Spry PG, et al: The Bristol Shared Care Glaucoma Study-validity of measurements and patient satisfaction. J Public Health Med 1997, 19:431-436.

4. Gray SF, Spry PG, Brookes ST, et al: The Bristol shared care glaucoma study: outcome at follow up at 2 years. Br J Ophthalmol 2000, 84:456-463.

5. Spencer IC, Spry PG, Gray SF, et al: The Bristol Shared Care Glaucoma Study: study design. Ophthalmic Physiol Opt 1995, 15:391-394.

6. Spencer IC, Coast J, Spry PGD, Smith L, Sparrow JM: The Cost of monitoring glaucoma patients by community optometrists. Ophthal Physiol Opt 1995, 15(5):383-386.

7. Lemij HG, Holtzer-Goor KM, Klazinga NS, Koopmanschap MA, Plochg T, Van Sprundel E: Monitoring of stable glaucoma patients: evaluation of the effectiveness and efficiency of a Glaucoma follow-up unit, staffed by nonphysician Health Care Professionals, as an intermediate step towards glaucoma monitoring in primary care. [http://www.imta.nl], (publications, reports, 2010)

8. Bourne RR, French KA, Chang L, Borman AD, Hingorani M, Newsom WD Can a community optometrist-based referral refinement scheme reduce false-positive glaucoma hospital referrals without compromising quality of care? The community and hospital allied network glaucoma evaluation scheme (CHANGES). Eye (Lond) 2010, 24(5):881-7.

9. Banes MJ, Culham LE, Crowston JG, Bunce C, Khaw PT: An optometrist's role of co-management in a hospital glaucoma clinic. Ophthalmic Physiol Opt 2000, 20(5):351-359.

10. Harper R, Radi N, Reeves BC, Fenerty C, Spencer AF, Batterbury M: Agreement between ophthalmologists and optometrists in optic disc assessment: training implications for glaucoma co-management. Graefes Arch Clin Exp Ophthalmol 2001, 239(5):342-50.

11. Harper R, Reeves B, Smith G: Observer variability in optic disc assessment: implications for glaucoma shared care. Ophthalmic Physiol Opt 2000, 20(4):265-273.

12. Oster J, Culham LE, Daniel R: An extended role for the hospital optometrist. Ophthalmic Physiol Opt 1999, 19(4):351-356.

13. Spry PG, Spencer IC, Sparrow JM, Peters TJ, Brookes ST, Gray S, Baker I, Furber JE, Easty DL: The Bristol Shared Care Glaucoma Study: reliability of community optometric and hospital eye service test measures. $\mathrm{Br} J$ Ophthalmol 1999, 83(6):707-12.
14. Hume J, Abbott F: Setting up a shared care glaucoma clinic. Nurs Stand 1995, 10(11):34-6.

15. Hitchings R: Shared care for glaucoma. Br J Ophthalmol 1995, 79(7):626.

16. Gelvin JB: Co-management of patients with glaucoma. Optom Clin 1994, 4(2):81-100.

17. Adams CM, Alexander LJ, Bartlett JD, Classé JG: Co-management of patients with glaucoma. Optom Clin 1992, 2(4):143-56

18. Azuara-Blanco A, Burr J, Thomas R, Maclennan G, McPherson S: The accuracy of accredited glaucoma optometrists in the diagnosis and treatment recommendation for glaucoma. Br J Ophthalmol 2007, 91(12):1639-43.

19. Oostenbrink JB, Bandel AP, Sol JCA, Van Ineveld BM: Kostprijsberekening voor bedrijfsvoering en economische evaluatie. Een vergelijking van methoden en resultaten. TSG 2000, 7:432-438.

20. Oostenbrink JB, Koopmanschap MA, Rutten F: Guidelines for cost calculation. Pharmacoeconomics 2002, 20(7):443-454.

21. Oostenbrink JB, Bouwmans CAM, Koopmanschap MA, Rutten FFH: Manual for costing research (in Dutch). Amstelveen: Health Care Insurance Board; 2004.

22. Druss BG, Marcus SC, Olfson M, Tanielian T, Pincus HA: Trends in care by Nonphysician Clinicians in the United States. N Engl J Med 2003, 348:130-7.

23. Lay JL, Murdoch JR: The rate of visual field loss in untreated primary open angle glaucoma. Br J Ophthalmol 1993, 77(3):176-178.

24. Broman AT, Quigley HA, West SK, et al: Estimating the Rate of Progressive Visual Field Damage in Those with Open-Angle Glaucoma, from CrossSectional Data. Invest Ophthalmol and Vis Sci 2008, 49:66-76.

\section{Pre-publication history}

The pre-publication history for this paper can be accessed here: http://www.biomedcentral.com/1472-6963/10/312/prepub

doi:10.1186/1472-6963-10-312

Cite this article as: Holtzer-Goor et al:: Cost-effectiveness of monitoring glaucoma patients in shared care: an economic evaluation alongside a randomized controlled trial. BMC Health Services Research 2010 10:312.

\section{Submit your next manuscript to BioMed Central and take full advantage of:}

- Convenient online submission

- Thorough peer review

- No space constraints or color figure charges

- Immediate publication on acceptance

- Inclusion in PubMed, CAS, Scopus and Google Scholar

- Research which is freely available for redistribution 